\title{
The Cochrane Collaboration: making the results of controlled trials properly accessible
}

\author{
Andrew Herxheimer
}

Cochrane Centre, Summertown Pavilion, Middle Way, Oxford OX2 7LG, UK

The Cochrane Centre in Oxford was officially opened in November 1992, and is perhaps the most visible part so far of the recently launched National Health Service Research and Development Programme. Its task is to facilitate and extend the creation of systematic reviews of randomized controlled trials (RCTs) evaluating health care. It is named after Archie Cochrane (1909-1988), the epidemiologist who first emphasized that reliable information from RCTs, together with other essential information, is vital for making sound decisions in health care and research. Most clinicians and clinical scientists who have tried to review the evidence for a particular procedure or treatment have felt frustrated by the difficulties of finding out what RCTs have been done, and of interpreting their results critically.

The UK Cochrane Centre is just one element of the rapidly evolving Cochrane Collaboration. Together with Cochrane Centres in Denmark and Canada and probably also in Australia, Italy and the United States, the Cochrane Collaboration will help to assemble and disseminate evidence derived from systematic reviews of RCTs. These differ from traditional reviews in that they are prepared as methodically and as meticulously as a piece of primary research, and they include a detailed description of the wav in which the trials were identified, selected and evaluated. Specifically, the Cochrane Collaboration is working to build and maintain a database of systematic, up-to-date reviews of RCTs of health care, and to make them readily accessible through electronic media.

The staff of the UK Cochrane Centre is small: the director, Iain Chalmers (who founded the National Perinatal Epidemiology Unit and edited the Oxford Database of Perinatal Trials), ${ }^{1}$ myself as a part-time consultant, a computer programmer, an information specialist, an editorial coordinator, an administrator and a secretary. The UK Cochrane Centre is linked with the Clinical Trial Service Unit

Correspondence: A. Herxheimer, F.R.C.P. Received: 5 July 1993 at Oxford University, the Department of Clinical Epidemiology and Biostatistics at McMaster University, and The Online Journal of Current Clinical Trials $^{2}$ published by the American Association for the Advancement of Science.

\section{How the Cochrane Centres are supporting the Collaboration}

The time needed to prepare valid reviews of RCTs tends to be grossly underestimated. Lack of experience and time often force good scientists to produce scientifically inadequate reviews. The Cochrane Centres will give practical support to those preparing and updating reviews within the Cochrane Collaboration in several ways.

Systematic reviews of RCTs must be based on as high a proportion of eligible studies as possible. In addition to a register and library of published reviews of RCTs, therefore, the UK Cochrane Centre is helping to coordinate the creation of as comprehensive as possible a register of RCTs, ${ }^{3}$ in collaboration with the US National Institutes of Health. Because bibliographic databases like MEDLINE identify only around $50 \%$ of RCTs, selected journals are being searched by hand. Efforts are meanwhile being made to improve the rate of RCT retrieval from bibliographic databases in future. The Register of RCTs will also aim to include references to unpublished, ongoing and planned controlled trials, so that people preparing systematic reviews can consider them.

In addition to containing completed reviews of RCTs, the Cochrane Database of Systematic Reviews will include details of reviews which are being prepared or planned. This information will help participants in the Cochrane Collaboration to avoid unnecessary duplication of effort, and others to know about forthcoming systematic reviews.

The Centres are developing protocols and software to help people preparing systematic reviews, and they collaborate to develop policies and set standards for this work, based when possible on relevant methodological research. 


\section{An editorial system based on collaborative review groups}

Those contributing reviews to the Cochrane Database do so as members of collaborative review groups, each coordinated by an editorial team which oversees a group of related reviews. These groups may be problem-based (for example, breast cancer), intervention-based (for example, nutrition) or specialty-based (for example, primary care). Reviewers considering whether to form such a review group can attend workshops to discuss some of the likely implications with already established groups. The group responsible for reviewing RCTs in pregnancy and childbirth now maintains about 600 systematic reviews of RCTs, prepared by over 30 reviewers in seven countries; it has to deal with 200-300 new reports of trials every year. The editorial team of this review group consists of four editors, an administrator and a data clerk.

The module of reviews of RCTs in pregnancy and childbirth is being used as the pilot to explore how best to develop a module-based system for building, updating and disseminating the Cochrane Database of Systematic Reviews. It is essential, for example, to make it easy for the reviews to be criticized and amended when necessary. The use of electronic publications, such as the Online Journal

\section{References}

1. Chalmers, I. Electronic publication of continuously updated overviews (meta-analyses) of controlled trials. ISDB Rev 1991, 1: $15-18$.

2. Information for authors and subscribers on The Online Journal of Current Clinical Trials is available from the Managing Editor in Washington, DC; fax: (202) 842-2868. of Current Clinical Trials, should greatly facilitate interaction between critics, authors and editors of $\frac{\not}{\infty}$ the reviews. As the Cochrane Database will be $\varrho$ updated and amended constantly, electronic media $\hookrightarrow$ offer obvious advantages for disseminating its $\vec{F}$ contents. The complete database will be distributed online and on CD-ROM; specialty databases for $\frac{}{0}$ particular groups of users will be compiled and $\overline{\bar{\omega}}$ then published on disk. The first of these, the $\overparen{\Phi}$

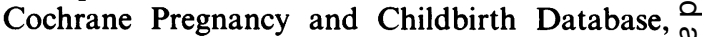
was published on a single $3 \frac{1}{2}$ inch disk in June $1993 .{ }^{4} \mathrm{c}$ It will be updated twice a year. A notable feature of $\vec{O}$ all the reviews on the disk is that the results of trials are displayed graphically as well as in tables.

The up-to-date systematic reviews of RCTs being prepared and maintained by the Cochrane Collaboration can help clinicians to keep their practice up to date and should facilitate the development of soundly based clinical guidelines. $\stackrel{\infty}{\vec{V}}$ They are also essential for ensuring that the lessons $\infty$ from previous studies are applied in the design of $\stackrel{\mathcal{V}}{ }$ new clinical trials. Because reviews are contributed 0 to the Cochrane Database on the understanding that copyright will not be assigned exclusively to $z$ any publisher, all journals, as well as various electronic media, can play their part in disseminating the results of these reviews, and thus help to ensure that the findings can be applied in practice

. Chalmers, I., Dickersin, K. \& Chalmers, T.C. Getting to grips with Archie Cochrane's agenda. Br Med J 1992, 305: 786-788. 4. The Cochrane Collaboration. Pregnancy and Childbirth 은 Database. Details from Cochrane Updates on Disk, Update $\mathrm{Q}$ Software Ltd, Manor Cottage, Little Milton, Oxford $\vec{\overrightarrow{ }}$ OX44 7QB, UK. Tel./fax: 0844278887. 